\title{
Gravitational collapse to toroidal, cylindrical and planar black holes gr-qc/9709013
}

\author{
José P. S. Lemos \\ Departamento de Astrofísica, Observatório Nacional-CNPq, Rua General José Cristino 77, 20921 Rio de Janeiro, Brazil E\} \\ Departamento de Física, Instituto Superior Técnico, Av. Rovisco Pais 1, 1096 Lisboa, Portugal.
}

\begin{abstract}
Gravitational collapse of non-spherical symmetric matter leads inevitably to non-static external spacetimes. It is shown here that gravitational collapse of matter with toroidal topology in a toroidal anti-de Sitter background proceeds to form a toroidal black hole. According to the analytical model presented, the collapsing matter absorbs energy in the form of radiation (be it scalar, neutrinos, electromagnetic, or gravitational) from the exterior spacetime. Upon decompactification of one or two coordinates of the torus one gets collapsing solutions of cylindrical or planar matter onto black strings or black membranes, respectively. The results have implications on the hoop conjecture.

PACS numbers: 04.20.Jb, 97.60.Lf.
\end{abstract}

\section{Introduction}

Black hole (BH) solutions in an anti-de Sitter (adS) background whose event horizons have planar [1], cylindrical [2], and toroidal topology [2,3] have been found recently (see also ical Schwarzschild BH has come from its role as the final state of complete gravitational collapse of a star, it is useful to investigate if these BHs with different topology may also emerge from gravitational collapse of some matter distribution.

An important feature of spherical collapse onto a Schwarzschild BH is that, due to Birkhoff's theorem, spacetime is static outside the matter and the collapse proceeds without emission of gravitational waves. The same is true for the collapse of spherical matter in an adS background, i.e., in a spacetime with negative cosmological constant. On the other hand, it is well known that the collapse of cylindrical systems proceeds with emission of gravitational waves [5 8] which creates additional problems in the modeling of these collapsing systems. It has also been known for a long time that collapsing infinite dust cylinders form naked singularities [5], not BHs. This violates the cosmic censorship conjecture [9] (which forbids the existence of singularities not surrounded by an horizon), but not the hoop conjecture [5] (which states that BHs form when and only when a mass $M$ gets compacted into a region whose circumference in every direction is less than its Scwharzschild circumference $4 \pi M$ $(G=C=1))$. The cosmic censorship is formulated to be applied to realistic gravitational collapse, which in principle does not include cylindrical matter. However, in a certain sense, cylindrical collapse can simulate the collapse of a finite spindle [10], near the central regions of the spindle the collapse behaves as if the spindle is an infinite cylinder. Besides these possible astrophysical applications, the collapse of infinite cylinders probes and tests the structure of general relativity.

In a previous work it has been conjectured by us [3] that, since there are known cylindrical BH solutions with a negative cosmological constant, collapse of cylindrical matter in a background with negative cosmological constant could form a cylindrical BH (i.e., a black string) rather than a naked singularity, violating in this way the hoop conjecture but not the cosmic censorship. In this work we show that indeed cylindrical BHs (or black strings) form from gravitational collapse of cylindrical matter distribution. As the azimuthal cylindrical coordinate can be decompactified this solution also shows that planar BHs (or black membranes) can also form form gravitational collapse of a planar distribution. Moreover, since the 'vertical' infinite cylindrical coordinate can, in turn, be compactified this solution also shows that toroidal BHs can form from gravitational collapse. Throughout this paper we will work mainly with the toroidal topology in mind, although the results can be modified straightforwardly to the other two cases, the main differences being the topologies themselves and the mass parameter which in the planar case is a surface mass density, in the cylindrical case is a linear mass density, and in the toroidal case it is a mass. In brief, we show that black membranes, black strings and toroidal BHs form from gravitational collapse.

Contrary to the spherical case, and as in the usual cylindrical collapse, the metric outside the collapsing toroidal (cylindrical or planar) matter is non-static. However, surprisingly, the problem can be solved exactly by using a modified Vaidya metric appropriate to the toroidal (cylindrical or planar) collapse (see section 2). Of course, this metric obeys Einstein field equations, and it describes the gravitational field associated with a toroidal (cylindrical or planar) flow of unpolarized scalar, neutrino, electromagnetic or gravitational radiation in the geometrical optics approximation. The interior solution we use is a modified Friedmann solution also applicable to toroidal (cylindrical or planar) topology (section 3). By making a smooth matching at the interface (section 4) we find that the flux of waves modeled by the modified Vaidya metric is an incoming flux, and consequently that the mass parameter of the collapsing matter grows up to the formation of the BH. By carefully choosing the right amout of incoming flux one avoids the emission of graviational waves from the collapsing matter. Finally, one can study the $\mathrm{BH}$ formation from the inside and outside points of view (section 5) and draw 
some conclusions (section 6).

\section{Exterior Radiating Solution}

As stated in the introduction, the Schwarzschild metric represents the external field of a collapsing spherical star, implying that in spherical collapse there is no emission of gravitational waves. Another spherically symmetric solution of Einstein field equations is the Vaidya metric which describes the gravitational field of an isotropic flow of unpolarized radiation in the geometrical optics approximation. It is usually employed in the study of imploding radiation shells [11], as well as in modeling the external field of radiating stars [12] and evaporating $\mathrm{BHs}$ [13. In the case of static or collapsing spherical stars, one can usually neglect the effects of this radiation and treat the external field as being given by the Schwarzschild metric. However, one should consider the Vaidya metric in the latest stages of the collapse when a supernova and a neutron star are formed accompanied by copious emission of neutrinos and photons. It is also possible that a $\mathrm{BH}$ forms directly from the collapsing star without passing through the strong radiative stage, in which case the Schwarzschild metric gives again an accurate description for the external field. If the star is slightly non-spherical, the latest stages of such a direct collapse onto a $\mathrm{BH}$ produce some gravitational waves which have to be treated as a perturbation on the Schwarzschild spacetime. The effects of adding a negative cosmological constant do not alter radically the description. The main difference would be that the outside spacetime should be described by the Schwarzschild-adS metric or when relevant by the Vaidya-adS metric.

The situation changes drastically for a star with nonspherical topology. The reason being that, since there is no analogue of Birkhoff's theorem, a collapsing nonspherical symmetric star produces a non-static external spacetime. Notwithstanding, as we will show, one can treat the problem analitically for the gravitational collapse of toroidal (cylindrical or planar) configurations.

The Einstein field equations are

$$
G_{a b}+\Lambda g_{a b}=8 \pi T_{a b},
$$

where $G_{a b}, g_{a b}, T_{a b}$ are the Einstein, the metric and the the energy-momentum tensors, respectively, and $\Lambda$ is the cosmological constant $(G=C=1)$. The equations admit the following solution

$$
d s^{2}=-\left(\alpha^{2} r^{2}-\frac{q m(v)}{\alpha r}\right) d v^{2}+2 d v d r+r^{2}\left(d \theta^{2}+d \phi^{2}\right)
$$

for an energy-momentum tensor given by

$$
\begin{aligned}
T_{a b} & =\frac{q}{8 \pi \alpha r^{2}} \frac{d m(v)}{d v} k_{a} k_{b}, \\
k_{a} & =-\delta_{a}^{v}, \quad k_{a} k^{a}=0 .
\end{aligned}
$$

Here $\alpha \equiv \sqrt{\frac{-\Lambda}{3}}, v$ is the advanced time coordinate with $-\infty<v<\infty, r$ is the radial coordinate with $0<r<\infty$, and $\theta, \phi$ are the coordinates which describe the two-dimensional zero-curvature space generated by the two-dimensional commutative Lie group $G_{2}$ of isometries. The topologies of this two-dimensional space can be (i) $T^{2}=S^{1} \times S^{1}$, the flat torus model $\left[G_{2}=U(1) \times U(1)\right]$, (ii) $R \times S^{1}$, the cylindricallly symmetric model $\left[G_{2}=R \times U(1)\right]$, and (iii) $R^{2}$, the planar model $\left[G_{2}=E_{2}\right]$. In the toroidal case we choose $0 \leq \theta<2 \pi$, $0 \leq \phi<2 \pi$, in the cylindrical case $-\infty<\theta<\infty$, $0 \leq \phi<2 \pi$, and in the planar case $-\infty<\theta<\infty$, $-\infty<\phi<\infty$. The parameter $q$ has different values depending on the topology of the two-dimensional space. For the torus $q=\frac{2 \alpha}{\pi}$ and $m(v)$ is a mass, for the cylinder $q=4$ and $m(v)$ is a mass per unit length, and for the plane $q=\frac{2}{2}$ and $m(v)$ is a mass per unit area.

Metric (2) is a modified Vaidya solution pertinent to toroidal (cylindrical or planar) topology. From the energy-momentum tensor given in equation (3) one can infer that it describes the gravitational field of a toroidal (cylindrical or planar) flow of unpolarized radiation in the geometrical optics approximation, and it can be used in modeling the external fields of matter with toroidal (cylindrical or planar) topology radiating or absorving energy. Besides modeling radiation in the form of neutrinos or electromagnetic waves, $T_{a b}$ in (3) can also represent scalar or gravitational radiation in an adequate limit. Now, noting that the energy-density of the radiation is $\epsilon=\frac{q}{8 \pi \alpha r^{2}} \frac{d m}{d v}$, one sees that the weak energy condition for the radiation is satisfied whenever $\frac{d m}{d v} \geq 0$, i.e., the radiation is imploding. For $m=$ constant one has vacuum and equation (2) describes a static toroidal (cylindrical or planar) black hole in ingoing (advanced time) EddingtonFinkelstein coordinates. The values of the parameter $q$ given above, were taken from ADM masses of the corresponding static BHs found in [1].

In these coordinates, lines with $v=$ constant represent incoming radial null vectors whose generator vectors have the form $k^{a}=(0,-1,0,0)$, with $k_{a}=(-1,0,0,0)$ (see equation (3)) . The generators $l^{a}$ of outgoing null lines do not have this simple form. By the conditions $l_{a} l^{a}=0$ and $l_{a} k^{a}=-1$ one finds $l^{a}=\left(1, \frac{1}{2}\left(\alpha^{2} r^{2}-\frac{q m(v)}{\alpha r}\right), 0,0\right)$. The equation for outgoing radial null geodesics $r(v)$ can easily be find by puting $d s^{2}=0$ in metric (2), yielding,

$$
\frac{d r}{d v}=\frac{1}{2}\left(\alpha^{2} r^{2}-\frac{q m(v)}{\alpha r}\right) .
$$

The exterior solution discussed here is to be matched to the interior metric presented in the following section.

\section{Interior Matter Solution}

We now assume that the interior spacetime is made of a homogeneous collapsing dust cloud whose spacetime is described through a flat Friedmann-Robertson-Walker type metric given by

$$
d s^{2}=-d t^{2}+a(t)^{2}\left(d l^{2}+l^{2}\left(d \theta^{2}+d \phi^{2}\right)\right),
$$

where $t$ and $l$ are comoving coordinates, and again, $\theta, \phi$ are the coordinates which describe the two-dimensional 
zero-curvature space (torus, cylinder or plane). Due to Einstein field equations (11) we have to set to zero the usual Friedmann-Robertson-Walker curvature parameter, $k=0$ without loss of generality. For dust the energymomentum tensor is given by

$$
T_{a b}=\rho u_{a} u_{b}
$$

where $\rho$ is the energy-density of the matter, and $u^{a}$ its four-velocity. Einstein field equations (11) yield

$$
\begin{gathered}
\frac{1}{a^{2}}\left(\frac{d a}{d t}\right)^{2}=\frac{8 \pi}{3} \rho-\alpha^{2} \\
\frac{1}{a} \frac{d^{2} a}{d t^{2}}=-\frac{1}{2 a^{2}}\left(\frac{d a}{d t}\right)^{2}-\frac{3 \alpha^{2}}{2}
\end{gathered}
$$

where again $\alpha \equiv \sqrt{\frac{-\Lambda}{3}}$. Integration of these equations give

$$
\rho=\frac{\rho_{0}}{\sin ^{2}\left(\frac{3}{2} \alpha t\right)}
$$

and

$$
a=a_{0} \sin ^{2 / 3}\left(\frac{3}{2} \alpha t\right)
$$

where $\rho_{0}$ and $a_{0}$ are constants, with $\rho_{0}=\frac{3 \alpha^{2}}{8 \pi}$. Restoring the constant $G$ one has $\rho_{0}=\frac{3 \alpha^{2}}{8 \pi G}$, which means the initial density is independent of the mass and radius of the initial configuration (a similar situation was found in 14 for gravitational collapse in Lovelock gravity). This could be expected, since for very small $\alpha$ one finds $\rho=\frac{1}{6 \pi G t^{2}}$, recovering the flat model with toroidal topology and (almost) zero cosmological constant, similar in its timedependence to the spherical flat-Friedmann model. Note also that $\rho a^{3}=$ constant. Defining $\bar{t} \equiv \frac{3}{2} \alpha t$, one sees that the solution is valid in the time range $0<\bar{t}<\pi$. From $0<\bar{t}<\pi / 2$ the matter is expanding, from $\pi / 2<\bar{t}<\pi$ the matter is collapsing. At $\bar{t}=\pi / 2$ there is a moment of time symmetry. We are interested in the collapsing part of the solution, and thus we take $\pi / 2 \leq \bar{t}<\pi$. The energy-density of the matter as well as the Kretschmann scalar blow up at $\bar{t}=\pi$, indicating the formation of a spacetime singularity.

\section{Matching}

To match the interior and exterior spacetimes, across an interface of separation $\Sigma$, we use the junction conditions

$$
\begin{aligned}
& \left.\left.d s_{+}^{2}\right]_{\Sigma}=d s_{-}^{2}\right]_{\Sigma} \\
& \left.\left.K_{a b}^{+}\right]_{\Sigma}=K_{a b}^{-}\right]_{\Sigma}
\end{aligned}
$$

where $K_{a b}$ is the extrinsic curvature,

$$
K_{a b}^{ \pm}=-n_{e}^{ \pm} \frac{\partial^{2} x_{ \pm}^{e}}{\partial \xi^{a} \partial \xi^{b}}-n_{e}^{ \pm} \Gamma_{c d}^{e} \frac{\partial x_{ \pm}^{c}}{\partial \xi^{a}} \frac{\partial x_{ \pm}^{d}}{\partial \xi^{b}}
$$

and $n_{e}^{ \pm}$are the components of the unit normal vector to $\Sigma$ in the coordinates $x_{ \pm}$, and $\xi$ represents the intrinsic coordinates in $\Sigma$. The subscripts \pm represent the quantities taken in the exterior and interior spacetimes. Both the metrics and the extrinsic curvatures in (11)-(12) are evaluated at $\Sigma$. It is useful to define a metric intrinsic to $\Sigma$ as

$$
d s_{\Sigma}^{2}=-d \tau^{2}+R^{2}(\tau)\left(d \theta^{2}+d \phi^{2}\right)
$$

where $\tau$ is the proper time on $\Sigma$.

We analyse first the surface $\Sigma$ as viewed from the exterior spacetime. To match the exterior metric with the metric on $\Sigma$ we use the junction condition (11) and metrics (2), (14), to obtain

$$
r(v)]_{\Sigma}=R(\tau)
$$

and

$$
\left.\left.\alpha^{2} r^{2}-\frac{q m(v)}{\alpha r}-2 \frac{d r}{d v}\right]_{\Sigma}=\frac{1}{(d v / d \tau)^{2}}\right]_{\Sigma}
$$

where both equations are evaluated on $\Sigma$. The unit normal to $\Sigma$ in the exterior spacetime is

$$
n_{e}^{+}=\frac{1}{\sqrt{-2 \frac{d r_{\Sigma}}{d v}+\alpha^{2} r_{\Sigma}^{2}-\frac{q m(v)}{\alpha r_{\Sigma}}}}\left(-\frac{d r_{\Sigma}}{d v}, 1,0,0\right) .
$$

Using (16) and $\frac{d r}{d v}=\frac{d r / d \tau}{d v / d \tau}$ we can put (17) in the form

$$
n_{e}^{+}=\left(-\frac{d r_{\Sigma}}{d \tau}, \frac{d v_{\Sigma}}{d \tau}, 0,0\right) .
$$

From now on, we will usually omit the subscript $\Sigma$ to denote evaluation at the interface. From (13) we then get the $K_{a b}^{+}$component of interest:

$$
K_{\theta \theta}^{+}=-r \frac{d r}{d \tau}+r \frac{d v}{d \tau}\left(\alpha^{2} r^{2}-\frac{q m(v)}{\alpha r}\right)
$$

valid on $\Sigma$, of course.

We now analyse the surface $\Sigma$ as viewed from the interior spacetime. To match the interior metric with the metric on $\Sigma$ we use the junction condition (11) and metrics (5), (14), to obtain

$$
l_{\Sigma} a(t)=R(\tau)
$$

and

$$
\frac{d \tau}{d t}=1
$$

valid on $\Sigma$. The unit normal to $\Sigma$ in the interior spacetime is 


$$
n_{e}^{-}=(0, a(t), 0,0)
$$

From (13) we then have

$$
K_{\theta \theta}^{-}=l_{\Sigma} a(t)=R(\tau)
$$

In order to have a smooth matching one imposes that the extrinsic curvatures (19) and (23) must be equal, $K_{\theta \theta}^{+}=K_{\theta \theta}^{-}$, yielding

$$
-r \frac{d r}{d \tau}+r \frac{d v}{d \tau}\left(\alpha^{2} r^{2}-\frac{q m(v)}{\alpha r}\right)=R(\tau),
$$

an equation valid on $\Sigma$. Using (24) and (16) we find

$$
\frac{d v}{d \tau}=\frac{1}{1-d R / d \tau}
$$

on $\Sigma$. Using now (24), (16), 25) and (7) we have

$$
m(v)=\frac{\alpha}{q}\left(\frac{8 \pi}{3} \rho R^{3}-R(\tau)\right) .
$$

Recalling from equations (9)-(10) that $\rho R^{3}=\rho_{0} R_{0}^{3}$ with $R=a(t) l_{\Sigma}$ (see equation (20)), and using (15) we find from 26 )

$$
m(v)=\frac{\alpha}{q}\left(\frac{8 \pi}{3} \rho_{0} R_{0}^{3}-r_{\Sigma}(v)\right),
$$

which gives the evolution of the mass with the external time $v$. Equation (27) is the most important result of the section. By (20), we know $R(\tau)=l_{\Sigma} a(t)$, and from (10) we have the evolution of the radius of the surface of the star with time $t$ (or $\tau$, since $d t=d \tau$ ). Then from (25) one gets $v=v(\tau)$, or its inverse $\tau=\tau(v)$. Since from equation (15) $r_{\Sigma}(v)=R(\tau)$, we find that $r_{\Sigma}(v)$ is a known function of $v$. Unfortunately, integration of 25) to find $v(\tau)$ cannot be performed analytically. However, since for collapse $\frac{d R}{d \tau}<0$ we find that $v$ increases monotonically with $\tau$ or $t$. Thus, as the collapse proceeds $r_{\Sigma}$ decreases from its maximum value $r_{\Sigma_{0}} \equiv r_{\Sigma}\left(v_{0}\right)=R_{0}$, where $v_{0}$ denotes the time at the onset of the collapse. Therefore, we have obtained the result that the mass of the cloud $m(v)$ increases during the collapse due to the incoming flux of the high frequency radiation, be it in the form of scalar, neutrinos, electromagnetic or gravitational waves.

It is convenient to define an initial mass $m_{0}$ in terms of $\rho_{0}$ and $r_{\Sigma_{0}}=R_{0}$ through the equation

$$
\frac{q m_{0}}{\alpha}=\frac{8 \pi}{3} \rho_{0} r_{\Sigma_{0}}^{3}-r_{\Sigma_{0}}
$$

Then equation (27) can be written as

$$
m(v)=m_{0}+\frac{\alpha}{q}\left(r_{\Sigma_{0}}-r_{\Sigma}(v)\right) .
$$

This equation will be used in the next section.

\section{Black Hole Formation}

To study $\mathrm{BH}$ formation we distinguish two situations, the inside and outside stories.

For the interior of the star we use metric (5) and study collapse in the range $\pi / 2 \leq \bar{t}<\pi\left(\bar{t} \equiv \frac{3}{2} \alpha t\right)$. At the onset of the collapse, at time $\bar{t}=\pi / 2$, there are no singularities. The singularity forms at $\bar{t}=\pi$ where the curvature scalars and the density blow up (see equation (10)). The appearance of an apparent horizon indicates the formation of a $\mathrm{BH}$. Here, the apparent horizon is defined as the boundary of trapped twotori (two-cylinders or two-planes) in spacetime. To find this boundary we look for two-tori (two-cylinders or twoplanes) $Y \equiv a(t) l=$ constant whose outward normals are null, i.e., $\nabla Y \cdot \nabla Y=0$, yielding $l_{\mathrm{AH}}=-\frac{1}{d a / d t}$. Using equation (10) this is,

$$
\frac{l_{\mathrm{AH}}}{l_{\Sigma}}=-\left(\frac{3}{8 \pi \rho_{0} R_{0}^{3} \alpha}\right)^{1 / 3} \frac{\sin ^{1 / 3} \frac{3}{2} \alpha t}{\cos \frac{3}{2} \alpha t} .
$$

The apparent horizon first forms at the surface of the star $l_{\mathrm{AH}}=l_{\Sigma}$. Thus, for given $\rho_{0}, R_{0}$ and $\alpha$ one can find from equation (30) the time $t$ at which the apparent horizon first forms.

For outside observers the descripition is different as they should use metric (2). If one looks for trapped twotori (two-cylinders or two-planes) whose outwards normals are null, $\nabla r \cdot \nabla r=0$, one now obtains the condition

$$
\alpha^{3} r_{\mathrm{AH}}^{3}=q m(v) \text {. }
$$

Differentiating equation (31) and puting it back in the metric (2) gives $d s^{2}=\frac{2 q}{3 \alpha^{3} r^{2}} \frac{d m(v)}{d v} d v^{2}+r^{2}\left(d \theta^{2}+d \phi^{2}\right)$. The sign of $d m(v) / d v$ decides on the character of the AH. Here $d m(v) / d v>0$. Thus, the apparent horizon is an unphysical spacelike surface, interior to the the surface of the matter where the metric (2) is not valid. Equation (31) is only valid at $v=v_{\mathrm{AH}}$, the time at which the apparent horizon forms.

We have a dynamic situation. As the matter collapses, the mass of the toroidal star increases. To find the radius at which the apparent horizon forms we equate equation (31) to (29) to obtain a cubic equation for $r_{\mathrm{AH}}$

$$
\alpha^{3} r_{\mathrm{AH}}^{3}+\alpha r_{\mathrm{AH}}-\left(q m_{0}+\alpha r_{\Sigma_{0}}\right)=0 .
$$

This equation has one real root. Defining $C, A$ and $B$ as

$$
C=q m_{0}+\alpha r_{\Sigma_{0}}
$$

$$
A=1 / 3 \sqrt{\frac{C}{2}+\sqrt{\frac{C^{2}}{4}+\frac{1}{27}}},
$$

and

$$
B=1 / 3 \sqrt{\frac{C}{2}-\sqrt{\frac{C^{2}}{4}+\frac{1}{27}}}
$$


the solution for $r_{\mathrm{AH}}$ can be written as

$$
\alpha r_{\mathrm{AH}}=A+B .
$$

One can check that both procedures (the inside and outside stories) give the same time $t$ (and $v$ ) for the formation of the apparent horizon. As an example we choose $r_{\Sigma 0}=\frac{1}{2}, \rho_{0}=\frac{5}{\pi}, \alpha=\frac{2}{3}, q m_{0}=\frac{7}{9}$. Then puting $l_{\mathrm{AH}}=l_{\Sigma}$ in (30) gives $t=2.51$. On the other hand, one gets from (36) $r_{\mathrm{AH}}=1.09$, which upon using (10) suitably gives again $t=2.51$.

There is an interesting situation in toroidal collapse. The initial configuration can collapse from a negative mass parameter $m_{0}$ into a $\mathrm{BH}$ with positive mass. To find the minimum possible mass that yields a $\mathrm{BH}$ we set

$$
m\left(v_{\mathrm{AH}}\right) \equiv m_{\mathrm{AH}}=0 .
$$

Puting this condition back in equation (29) implies

$$
r_{\mathrm{AH}} \equiv r\left(v_{\mathrm{AH}}\right)=r_{\Sigma 0}+\frac{q}{\alpha} m_{0} .
$$

Equations (38) and (32) then give $r_{\mathrm{AH}}=0$. Thus, $m_{\mathrm{AH}}=0$ implies $r_{\mathrm{AH}}=0$. In addition, equation (38) gives that the initial mass should obey

$$
m_{0} \geq-\frac{\alpha}{q} r_{\Sigma 0}
$$

Below this value of $m_{0}$ the collapsing matter forms a naked singularity rather than a $\mathrm{BH}$.

To deal with the cubic equation (32) one can study two limiting situations. First, assume $C=q m_{0}+\alpha r_{\Sigma_{0}}<<1$ with $\alpha r_{\Sigma_{0}}>>m_{0}$. Then the solution is

$$
\alpha r_{\mathrm{AH}}=\alpha r_{\Sigma 0}\left(1-\alpha^{2} r_{\Sigma_{0}}^{2}+\frac{q m_{0}}{\alpha r_{\Sigma 0}}\right)
$$

In order that there is no black hole at the onset of the collapse one has $\alpha^{2} r_{\Sigma_{0}}^{2}>\frac{q m_{0}}{\alpha r_{\Sigma 0}}$, which implies $r_{\mathrm{AH}}<r_{\Sigma 0}$ always.

Second, if we assume $C=q m_{0}+\alpha r_{\Sigma 0}>>1$ with $\alpha^{3} r_{\Sigma 0}>C$ then the solution for the apparent horizon is

$$
\alpha r_{\mathrm{AH}}=\left(q m_{0}+\alpha r_{\Sigma_{0}}\right)^{1 / 3}-\frac{1}{3\left(q m_{0}+\alpha r_{\Sigma_{0}}\right)^{1 / 3}},
$$

where again $r_{\mathrm{AH}}<r_{\Sigma 0}$, as it should.

We still have to check that the collapsing star fades from sight to external observers. A null geodesic emerging form the surface of the star $r_{\Sigma}$ at a time $v$ arrives at an observer at point $r$ at time $v_{\text {obs }}$ given by

$$
v_{\mathrm{obs}}=v+\int_{r_{\Sigma}}^{r} \frac{d v}{d r} d r
$$

Using equation (4) this gives

$$
\begin{gathered}
v_{\mathrm{obs}}=v+\frac{2}{\alpha} \frac{1}{(q m(v))^{1 / 3}}\left[\frac{1}{6} \ln \left(\alpha r-(q m(v))^{1 / 3}\right)^{2}-\right. \\
\frac{1}{6} \ln \left(\alpha^{2} r^{2}+\alpha r(q m(v))^{1 / 3}+(q m(v))^{2 / 3}\right) \\
\left.+\frac{1}{\sqrt{3}} \tan ^{-1} \frac{2 \alpha r+(q m(v))^{1 / 3}}{\sqrt{3}(q m(v))^{1 / 3}}\right]_{r_{\Sigma}}^{r} .
\end{gathered}
$$

Then $v_{\text {obs }} \rightarrow \infty$ as the surface of the star approaches the apparent horizon, $r_{\Sigma} \rightarrow \frac{q m\left(v_{\mathrm{AH}}\right)}{\alpha}$. As seen by outside observers the collapse to a $\mathrm{BH}$ takes an infinite time. One can also check that the redshift $z$ of the light diverges as the apparent horizon is approached, $z \propto-\frac{d r_{\Sigma} / d t}{r_{\Sigma}-(q m)^{1 / 3}}$, implying that light from star gets ever dimmed. The solution (2) will then tend to the static $\mathrm{BH}$ solutions found in [1] 3 .

\section{Conclusions}

We have found collapsing solutions of toroidal (cylindrical, or planar) matter onto toroidal (cylindrical, or planar) BHs. In the model used there is a flux of high frequecy radiation towards the star. This incoming radiation has to be chosen with the right flux (see equation (27)) in order to avoid the emission of gravitational waves. If there is no such precisely chosen incoming flux, the toroidal (cylindrical or planar) configuration will presumably collapse to form a $\mathrm{BH}$ with emission of large amounts of gravitational waves 15 .

For external observers, the solution tends asymptotically with time to static $\mathrm{BH}$ solutions. The maximally analytical extension of these static BH solutions show unphysical regions, such as the white hole region behind the past event horizon, which also appear in the Schwarzschild solution. When one performs complete gravitational collapse of some matter configuration in an approprite background, this part of the solution will cover the unphysical regions of the maximally extended black hole. In the corresponding Penrose diagram, each point representing a torus, a cylinder or a plane, depending on the topology chosen, one visualizes collapsing solutions of toroidal, cylindrical or planar matter onto black holes, black strings or black membranes, respectively. Collapse to $\mathrm{BH}$ solutions with pseudospherical horizons have also been recently studied [16].

A further extension of this paper could be the inclusion of the Teichmuller complex parameter, which specifies conformally equivalent classes of the torus, into the metric of the toroidal BH [17] and see if it produces significant changes.

The Vaidya metric is also used to study the formation of naked singularities in spherical gravitational collapse [11]. In a preliminary study [18] we have found that collapse of toroidal (cylindrical or planar) radiation using the modified Vaidya metric of section 2 does not yield naked singularities, strengthening the claim made in the introduction that non-spherical collapse in a negative cosmological constant background may violate the hoop but not the cosmic censorship conjecture. 
[1] J. P. S. Lemos, Class. Quantum Gravity 12, 1081 (1995).

[2] J. P. S. Lemos, Phys. Lett. B 352, 46 (1995).

[3] J. P. S. Lemos, V. T. Zanchin, Phys. Rev. D 54, 3840 (1996).

[4] C. G. Huang, C. B. Liang, Phys. Lett. A 201, 27 (1995); R. G. Cai, Y. Z. Zhang, Phys. Rev. D 54, 4891 (1996); D. R. Brill, J. Louko, P. Peldán, gr-qc/9705012.

[5] K. S. Thorne, in Magic without Magic, ed. J. R. Klauder, Freeman and Company (1972), p. 231.

[6] T. Piran, Phys. Rev. Lett. 41, 1085 (1978); F. Echeverria, Phys. Rev. D 47, 2271 (1993).

[7] T. A. Apostolatos, K. S. Thorne, Phys. Rev D 46, 2435 (1992).

[8] K. Nakamura, H. Ishihara, "Cylindrical Domain Walls and Gravitational Waves", gr-qc/9710078.

[9] R. Penrose, Riv. Nuovo Cimento 1 (Numero Special), 252 (1969).

[10] S. L. Shapiro, S. A. Teukolsky, Phys. Rev. Lett. 66, 944 (1991).

[11] P. S. Joshi, Global Aspects in Gravitation and Cosmology, (Clarendon Press, Oxford, 1993).

[12] R. W. Lindquist, R. A. Schwartz, C. W. Misner, Phys. Rev., 137, B1364 (1965).

[13] J. W. York, in Quantum Gravity, ed. S. Christensen, (1983).

[14] A. Ilha, J. P. S. Lemos, Phys. Rev. D 55, 1788 (1997).

[15] I thank the referee for clarifying this point.

[16] S. Åminneborg, I. Bengtsson, S. Holst, P. Peldán, Class. Quantum Grav. 13, 2707 (1996); R. Mann, W. Smith, gr-qc/9703007.

[17] L. Vanzo, gr-qc/9705004.

[18] J. P. S. Lemos, "Collapse of cylindrical, toroidal or planar shells of radiation and the hoop and cosmic censorship conjectures", in preparation. 